Onkologie 1984;7:141-144

\title{
Advert
}

Am Ort der Infektion muß ein Antibiotikum wirken

Breites Spektrum, höchste Aktivität gegen Problemkeime vereinigt mit besten pharmakokinetischen Eigenschaften: FORTUM ${ }^{\circledR}$ (Ceftazidim).

Gute in vitro Ergehnisse eines Antibiotikums allein garantieren noch keinen klini-schen Erfolg. Ein Antibiotikum wird nur erfolgreich sein, wenn es in ausreichender Konzentration - ein entsprechendes antibakterielles Spektrum vorausgesetzt - auch an den Ortderlnfektiongelangt. Deshalbsindnebenderantimikrobiellen Wirksam-keit pharmakokinetische Eigenschaften ein wichtiges Beurteilungskriterium eines Antibiotikums.

Anzeige 2

Die wichtígsten pharmakokinetischen Parameter von Ceftazidim

Serumspitzen-konzentration nach 2g i.v. Bolus 192,8 mcg/ml

171 Min.

ca. $10 \%$

21,11

$4,51 / \mathrm{h}$

Serumhalbwertzeiten: (bei Patíenten)

Eiweißbindung

Verteilungsvolumen

Gesamtclearance

$>90 \%$

Urin-Recoveiy nach 12 Stunden

min. Zeit

25 Liter

i $1 \quad 1 \quad 1$ i i i i i i i i

0102030405060708090100

(Durchschnittswerte) \%

In der letzten Folge dieser Serie wurde besonders auf die antibakterielle Aktivität des neuen

Breitsprektrum-Cephalosporins FORTUM (Ceftazidim) eingegangen:

sein breites Spektrum

seine hohe Aktivität gegen Problemkeime wie indolpositive Proteus, Enterobacter oder Serratia seine extrem gute Wirksamkeit gegen Pseudomonas aeruginosa „Es (Ceftazidim) entfaltet eine hohe Aktivität gegen Pseudomonas aeruginosa und schließt damit eine Wir-kungslücke des Latamoxefs und Cefotaxims"

(H. Baumgärtner et al., 1983)

- $\quad$ seine hohe B-Laktamase-Stabilität

machen Ceftazidim schon zu einem 
Präparat, das mit besonderem Interesse

erwartet wird.

Wie steht es nun urn die pharmakokinetischen Eigenschaften von FORTUM?

Sie stehen der antibakteriellen Wirksamkeit der Substanz nicht nach:

Ceftazidim erreicht schnell hohe und langanhaltende Serum- und Gewebe-spiegel. Die

Serumhalbwertszeit bei Patíenten liegt bei etwa drei Stunden. Die Substanz zeichnet sich durch eine niedrige Eiweißbindung, ein großes Verteilungsvolumen und eine fastvollstän-dige renale (glomeruläre) Exkretion aus. Ceftazidim wird nicht metabolisiert und aufgrund der niedrigen biliären Ausscheidung ist die Darmbelastung gering.

Serumhalbwertzeit bei Patíenten nach i.v. Applikation

Mezlocillin

(Queue: nach Wittmann, D. H.: FAC 1-1, (1982), 175-184 u.FAC 2-3, (1983), 49-60

Cefoxiün

Niedrige Eiweißbindung, hohes Verteilungsvolumen und hohe renale Ausscheidung von

FORTUM entsprechen den Vorstellungen der Kliniker von einem modernen Cephalosporin.

Urinausscheidung

Eiweißbindung

Ceftazidim

Cefsulodin

Latamoxef

Cefotaxim

Ceftazidim Cefsulodin Latamoxef Cefotaxim

Cefoperazon

Cefoperazon

Ceftriaxon

Ceftriaxon

\section{I I I I I I I I I I}

0102030405060708090100

(Durchschnittswerte) \%

Anzeige

Zeitspanne, in de $\Gamma$ die MHI $1 / 8$-Werte von Pseudomonas aeruginosa und Staph. aureus im Serum überschritten werden.

Von besonderer Bedeutung flir den Kliniker ist die Zeitspanne, in der die Serumspiegel eines Antibiotikums über den MHK-Werten der relevanten Erre-ger liegen. Diese Daten erlauben Rück-schlüsse auf Dosierungsintervall und potentiellen Therapieerfolg. Für Ceftazi-dim wurden in der Tabelle die MHK90-

Werte(!) in Relation zu den erreichten Serumspiegeln gesetzt

Konzentration $(\mathrm{mcg} / \mathrm{ml})$

200

100

50

20

Die Serumspiegel liegen so lange so hoch, daß auch die gegen alle modernen Cephalosporine in vitro nur mäßig sensi-blen Staphylokokken in vivo (nachge-wiesenermaßen) gut erfaßt werden. 10

Ceftazidim 
Piperacillin

Gewebespiegel von Ceftazidim

Gewebe/ Köperflüssigkeit

Dosis und Applikation

Konzentration $(\mathrm{mcg} / \mathrm{ml})$

Zeit nach Verabr.

Hautblasen-

flüssigkeit

(Prob.)

1 g i.v.

44,7

$\mathrm{lh}$

Knochen, org.

2 g i.v.

31,1

$40 \mathrm{mi}$

Prostatagewebs-flüssigkeit

2 g i.v.

25,6

2

12,7

2

Skelettmuskel

2 g i.v.

9,4

2

FORTUM

$3 \mathrm{db} \omega<v 1 / 8 \mathrm{c}$

ãë

Latamoxef Cefotaxim Piperacillin

FORTUM

Latamoxef

Cefotaxim

-í i 1

101112 Std. Zeit

Ceftazidim verbindet hohe Wirksamkeit mit vorbildlicher Verträglichkeit

Die relevanten Kriterien für die Bei teilung der Verträglichkeitneuer Antibí tika sind neben biochemischen Lab $<$ parametern, vor allem die Allergis rungsrate und die Nierenverträglichk $\phi$ eventuelle Antabuseffekte oder ei Beeinträchtigung der Blutungszeit

Ceftazidim weist eine gute allgemei Verträglichkeit auf, verändert die Pi thrombinzeiten oder Blutungszeit nicht und ein Antabuseffekt ist ai grund der chemischen Struktur d Substanz nicht zu erwarten.

(Quelle: verändert nach Wittmann, D. H.: FAC 1-1, (1982), 175-184 und FAC 2-3, (1981), $467-$ 79 und Knothe, H, Dette, G.A.: J. Antimicrob. Chemother. (1981), 8 (Suppl. B), 33-41) 
$2 \mathrm{x} 2 \mathrm{~g}$ Ceftazidim pro die bei FORTUM versus 2er-Kombination (Gentamidn + Cefotaxim) schweren Infektionen $(<1000$ Neutrophiie/mm3)

Die normale Dosierung von FORTUM beträgt $2 \times 2 \mathrm{~g} /$ die auch bei schweren Infektionen. Bei Infektionen, bei denen $\Lambda$ ntibiotika generell höher dosiert werden, können $2 \mathrm{~g}$ FORTUM alle 8 Stunden gegeben werden. Patienten mit Mukoviszidose haben bis zu 9g/ die gut vertragen. Auch schwere Harn-wegsinfektionen reagieren im allgemei-nen gut auf 2 x 1 g Ceftazidim pro die. Bei Patienten mit eingeschränkter Nieren-funktion muß die Dosierung dem Grad der Einschränkung angepasst werden. Insgesamt beträgt die Regeldosis aber $2 \times 2 \mathrm{~g} /$ die - schwere Infektionen eingeschlossen.

Die Ergebnisse bei insgesamt ca. 2.600 Patienten

Ergebnisse der klinischen Studien

Entscheidend fur die Beurteilung eines neuen Antibiotikums sind neben dem antibakteriellen Spektrum und der Pharmakokinetik insbesondere die Ergebnisse der klinischen Prüfungen. Für Ceftazidim liegen derzeit die Daten von mehr als 4.000 Patienten vor. Die Ergebnisse dieser Studien geben zu den besten Hoffnungen für den Routineein-satz bei schweren und lebensbedrohen-den Infektionen Anlaß: auch die Thera-pieresultate bei schwersten Infektionen und bei immunsupprimierten Patienten wurden ausgesprochen positiv von den Untersuchern bewertet Nebenwirkun-gen waren selten, das Auftreten resisten-ter Stämme sogar sehr selten. In der Mehrzahl der Fälle konnte Ceftazidim als Monotherapie eingesetzt werden.

Nur bei einigen Fallen - meist bei neu-tropenischen Patienten - wurde es kom-biniert, um eine noch größere Therapie-sicherheit zu erlangen. Viele Studien belegen aber, daß durch eine Monotherapie mit Ceftazidim ebenso gute Thera-pieresultate erzielt wurden wie bei der Therapie mit der Kombination eines modernen Cephalosporins mit einem Aminoglykosid oder Ureidopenicillin. Ceftazidim wurde im Rahmen der klinischen Studien meist mit 3xlg oder 2x2g pro Tag gegeben (bei schweren Infektionen im Schnitt 3,7g/die). 